\title{
A Foxo/Notch pathway controls myogenic differentiation and fiber type specification
}

Tadahiro Kitamura, ${ }^{1,2}$ Yukari Ido Kitamura, ${ }^{1}$ Yasuhiro Funahashi, ${ }^{3}$ Carrie J. Shawber, ${ }^{3}$ Diego H. Castrillon, ${ }^{4}$ Ramya Kollipara, ${ }^{5}$ Ronald A. DePinho, ${ }^{5}$ Jan Kitajewski, ${ }^{3}$ and Domenico Accili ${ }^{1}$

\begin{abstract}
1Department of Medicine, Columbia University College of Physicians and Surgeons, New York, New York, USA. 2Metabolic Signal Research Center, Institute for Molecular and Cellular Regulation, Gunma University, Maebashi, Japan. ${ }^{3}$ Department of Pathology and Obstetrics/Gynecology, Columbia University College of Physicians and Surgeons, New York, New York, USA. ${ }^{4}$ Department of Pathology, University of Texas Southwestern Medical Center, Dallas, Texas, USA. ${ }^{5}$ Center for Applied Cancer Science, Departments of Medical Oncology, Medicine, and Genetics, and Belfer Institute for Innovative Cancer Science, Dana-Farber Cancer Institute, Harvard Medical School, Boston, Massachusetts, USA.
\end{abstract}

\begin{abstract}
Forkhead box $\mathrm{O}$ (Foxo) transcription factors govern metabolism and cellular differentiation. Unlike Foxodependent metabolic pathways and target genes, the mechanisms by which these proteins regulate differentiation have not been explored. Activation of Notch signaling mimics the effects of Foxo gain of function on cellular differentiation. Using muscle differentiation as a model system, we show that Foxo physically and functionally interacts with Notch by promoting corepressor clearance from the Notch effector Csl, leading to activation of Notch target genes. Inhibition of myoblast differentiation by constitutively active Foxo1 is partly rescued by inhibition of Notch signaling while Foxo1 loss of function precludes Notch inhibition of myogenesis and increases myogenic determination gene (MyoD) expression. Accordingly, conditional Foxo1 ablation in skeletal muscle results in increased formation of MyoD-containing (fast-twitch) muscle fibers and altered fiber type distribution at the expense of myogenin-containing (slow-twitch) fibers. Notch/Foxo1 cooperation may integrate environmental cues through Notch with metabolic cues through Foxo1 to regulate progenitor cell maintenance and differentiation.
\end{abstract}

\section{Introduction}

A central issue in regenerative medicine is understanding how highly specialized cell types arise from undifferentiated stem or progenitor cells (1). Germane to this issue is how biochemical signals engendered by microenvironmental and endocrine/nutritional cues are transcriptionally integrated to activate cellular differentiation processes.

The $\mathrm{O}$ subfamily of forkhead (Fox) proteins regulates hormonal, nutrient, and stress responses to promote cell survival and metabolism. The ability to fine-tune Foxo transcription is essential to controlling these cellular functions and is largely dependent on posttranscriptional modifications, including phosphorylation and acetylation (2). In addition to their role in terminally differentiated cells, Foxo proteins have also been implicated in myoblast (3), preadipocyte (4), and endothelial cell differentiation (5). Moreover, Foxo4 regulates vascular smooth muscle cell differentiation through interactions with myocardin (6). Foxo3 knockout mice display premature ovarian failure, consistent with a role for this gene in ovarian follicle maturation (7). The mechanisms by which Foxo proteins control cellular differentiation remain unclear, and recent conditional ablation studies are consistent with a significant degree of functional overlap among the 3 Foxo isoforms in the hematopoietic lineage $(8,9)$.

The Notch pathway plays an important role in neural, vascular, muscular, and endocrine differentiation during embryogenesis

Nonstandard abbreviations used: ChIP, chromatin immunoprecipitation (assay); DBD, DNA binding deficient; Foxo, forkhead box O; GST, glutathione-S-transferase; Hes, Hairy and Enhancer of split; Hey, Hes-related; Maml1, mastermind-like 1; Myf5, myogenic factor 5; MyoD, myogenic determination gene; Myog, myogenin; NcoR, nuclear corepressor; Notch1-IC, constitutively active Notch1; NTD, $\mathrm{NH}_{2}$ terminal domain; Pgc1 $\alpha$, Ppary coactivator $1 \alpha$; Smrt, silencing mediator for retinoid and thyroid hormone receptor.

Conflict of interest: The authors have declared that no conflict of interest exists. Citation for this article: J. Clin. Invest. 117:2477-2485 (2007). doi:10.1172/JCI32054
(10). Upon ligand-induced cleavage, the intracellular domain of the Notch receptor translocates to the nucleus, where it interacts with the DNA-binding protein Csl, changing its transcriptional properties from a suppressor to an activator of transcription (11). Csl targets include the Hairy and Enhancer of split (Hes) and Hesrelated (Hey) genes. Hes1 controls gut endoderm (12), preadipocyte (13), and neurogenic differentiation (14). Active Notch signaling, or Notch1 receptor gain of function, inhibits differentiation of $\mathrm{C} 2 \mathrm{C} 12$ and $10 \mathrm{~T} / 2$ myoblasts by suppressing myogenic determination gene $(M y o D)$ transcription (15-21).

It is noteworthy that Foxo1 gain of function (3-5) phenocopies Notch1 activation $(13,17,22,23)$ in every cellular differentiation context. Moreover, Foxo1 ablation (24) phenocopies Notch1 ablation (25) in mice. Despite these intriguing similarities, Foxo and Notch signal through 2 seemingly distinct mechanisms, the phosphatidylinositol 3-kinase pathway (Foxo) and the Hes/Hey pathway (Notch). In this study, we show that Foxo physically and functionally interacts with Notch by promoting corepressor clearance from Csl, thus controlling the myogenic program.

Myogenic precursors arise from mesodermal stem cells (26) and are converted into myotubes by a multistep process culminating in the expression of myogenic transcription factors of the myogenic regulatory factor (MRF) family (MyoD, myogenin, MRF4, and myogenic factor 5 [Myf5]) (27). Myogenic transcription factors heterodimerize with $\mathrm{E}$ proteins and promote expression of muscle-specific genes, acting in close coordination with myocytespecific MEF2 enhancer factors (28).

Adult muscle is a heterogeneous tissue, primarily defined by its myofiber content (29). Different myosin heavy chain (MyHC) subtypes characterize different myofibers. Type I fibers express primarily slow-twitch $\mathrm{MyHC}$ whereas type II fibers express fasttwitch MyHC (29). The process of fiber-type specification is controlled at multiple steps. First, there appears to be heterogeneity 

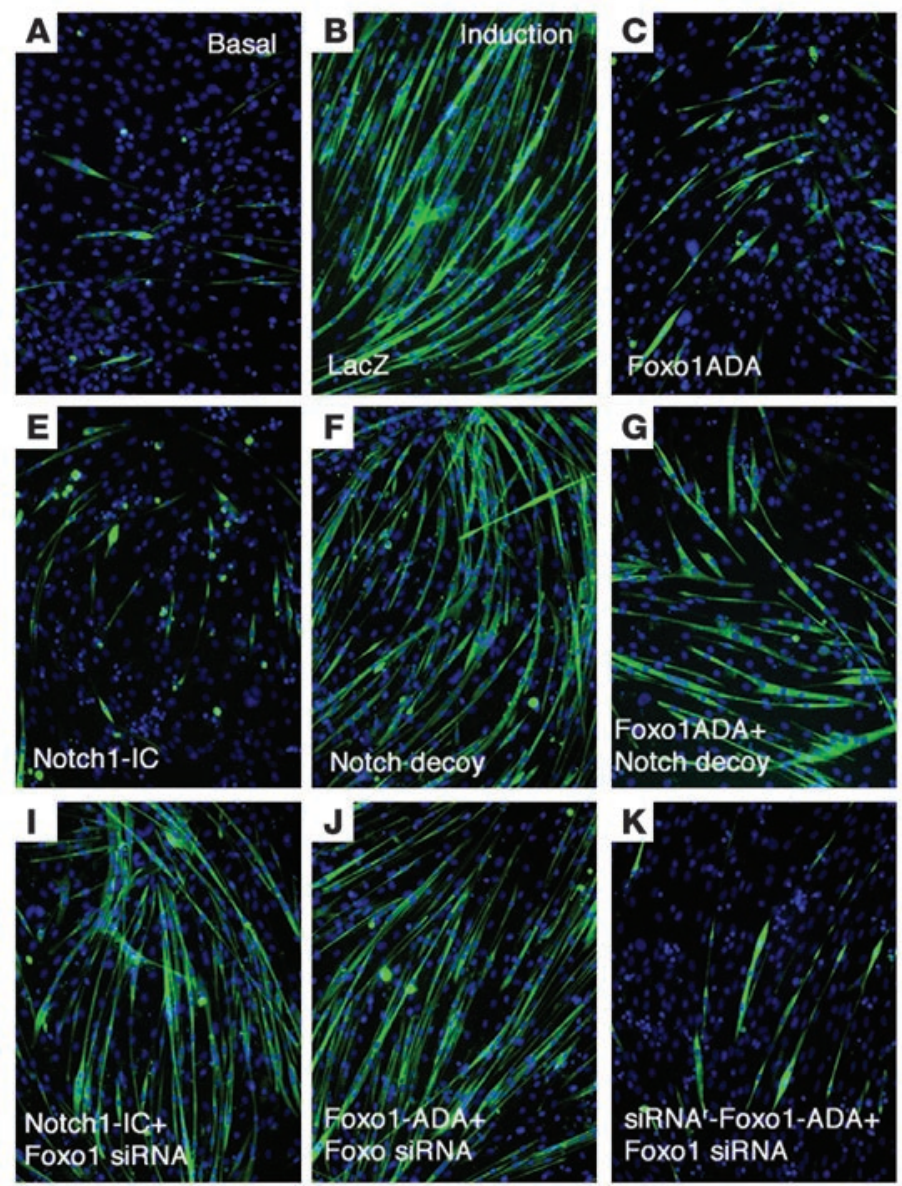

Figure 1

Regulation of myoblast differentiation by Foxo and Notch. C2C12 cells were immunostained with anti-myosin antibody (green) and DAPI (blue). See text for panel description. Each experiment was repeated at least 6 times. Original magnification, $\times 10$.

among myogenic precursor cells, and evidence from avian embryo cross-transplantation experiments indicates that early precursors contribute primarily to slow muscle fibers and later precursors to fast fibers (29). Postnatally, fiber type specification is also affected by cell autonomous factors, including innervation and endocrine/ nutritional cues (28). The Foxo coactivator Ppary coactivator $1 \alpha$ $(\mathrm{Pgc} 1 \alpha)$ plays a critical role in promoting the formation of slowtwitch fibers (30), and recent data have also implicated the Foxo deacetylase Sirt 1 in this process (31). Using conditional mutagenesis in mice, we show that Foxo1's role in suppressing MyoDdependent myogenesis in C2C12 cells is mirrored by an increase of MyoD-containing myofibers in Foxo1-deficient skeletal muscle, consistent with a key function in myoblast lineage specification.

\section{Results}

Interaction of Foxo1 and Notch signaling in C2C12 differentiation. To understand whether Notch and Foxo interact to control muscle development, we used a cellular differentiation model. C2C12 cells undergo myogenic conversion and myotube fusion upon growth factor withdrawal, a process associated with Foxo1 nuclear translocation (3). Accordingly, transduction of adenovirus encoding a constitutively active Foxo1 mutant (Foxo1-ADA; a mutant Foxo1 with the following amino acid substitutions: T24A, S253D, and
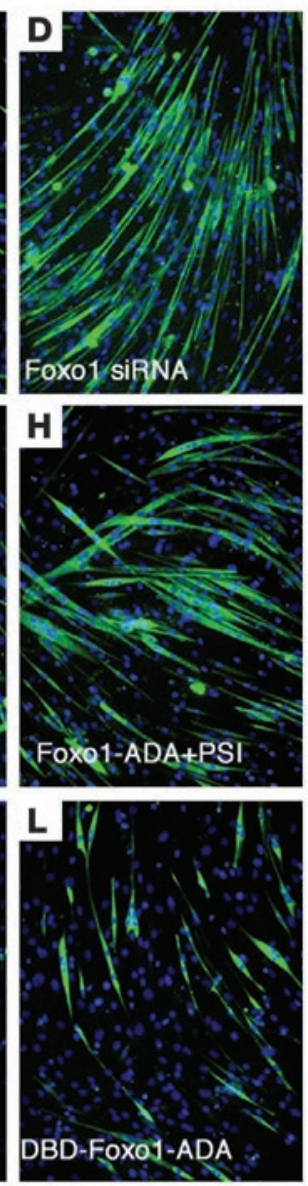

S316A) (4) blocked the effect of serum withdrawal to induce $\mathrm{C} 2 \mathrm{C} 12$ differentiation, as reflected by inhibition of myoblast fusion (Figure 1, A-C). Conversely, Foxo1 inhibition by siRNA did not affect these processes (Figure 1D). Similarly, constitutively active Notch 1 (Notch1-IC) phenocopied Foxo1-ADA in blocking myoblast differentiation (Figure 1E). Virtually all cells became transduced with the adenoviruses (Supplemental Figure 1; supplemental material available online with this article; doi:10.1172/JCI32054DS1). Foxo1 siRNA effectively suppressed expression of both endogenous Foxo1 and transfected FLAG-Foxo1 (Supplemental Figure 2) in a dose-dependent manner, without affecting control proteins or other Foxo isoforms (Supplemental Figure 3). Neither Foxo1-ADA nor Notch1-IC affected C2C12 proliferation (Supplemental Figure 4).

We asked whether we could preempt the effect of Foxo1-ADA by inhibition of endogenous Notch signaling. To this end, we used a truncated Notch 1 receptor lacking the transmembrane anchor and intracellular domain, which acts as a decoy receptor by binding Notch ligands $(32,33)$ (our unpublished observations). The decoy did not affect C2C12's ability to undergo differentiation in response to growth factor withdrawal (Figure $1 \mathrm{~F}$ ) but partly rescued Foxo1-ADA inhibition of myoblast differentiation (Figure 1G). As an alternative probe to block Notch signaling, the presenilin inhibitor (PSI) compound E (34) also rescued Foxo1-ADA inhibition of myoblast differentiation (Figure $1 \mathrm{H}$ ).

To examine the effect of Foxo1 on Notch signaling, we cotransfected Foxo1 siRNA and Notch1-IC. Foxo1 siRNA rescued inhibition of myoblast differentiation and myosin expression by Notch1-IC (Figure 1I) while control siRNA had no effect (data not shown). To rule out nonspecific effects of Foxo1 siRNA on myoblast differentiation, we generated an siRNA-resistant Foxo1-ADA (Supplemental Figure 5). Foxo 1 siRNA reversed the effects of Foxo1-ADA (Figure 1J) but failed to rescue inhibition of $\mathrm{C} 2 \mathrm{C} 12$ differentiation caused by siRNA-resistant Foxo1-ADA (Figure $1 \mathrm{~K}$ ). We present a quantitative analysis of these data in Figure 2A, showing that Foxo1 and Notch1-IC decreased myosin levels by more than $80 \%$ while Notch decoy and Foxo1 siRNA restored them to approximately $70 \%$ of fully differentiated cells. We obtained similar data by performing a morphometric analysis of myosin-positive cells (Figure 2B). These data indicate that Foxo1 is required for the effect of Notch on myoblast differentiation.

We next determined whether Foxo1 affects differentiation via its transcriptional function. To this end, we generated a DNAbinding deficient (DBD) mutant in the backbone of the ADA mutant by replacement of N208A and H212R (DBD-Foxo1-ADA) 
A

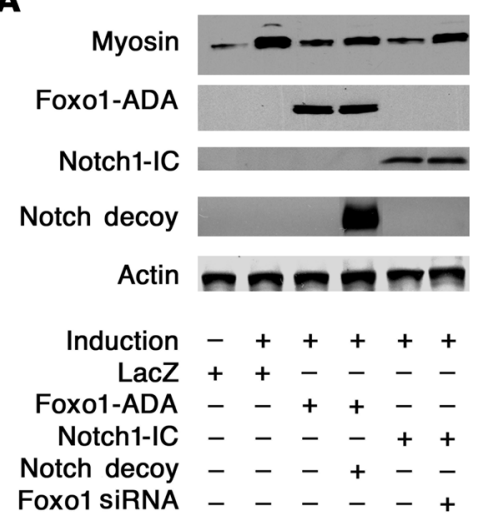

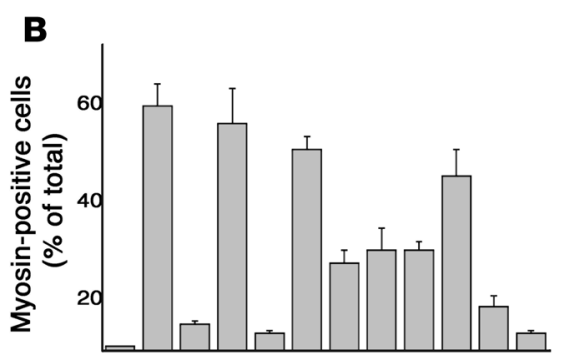

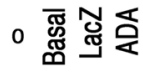

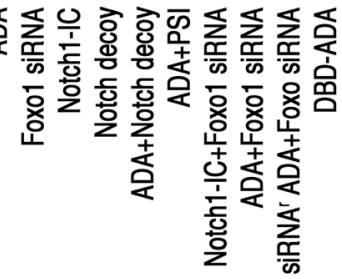

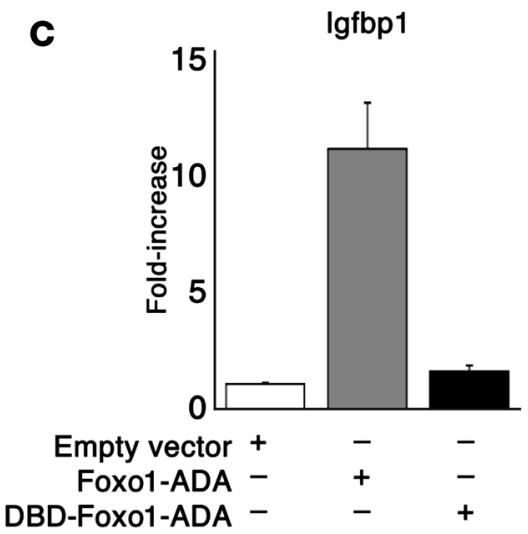

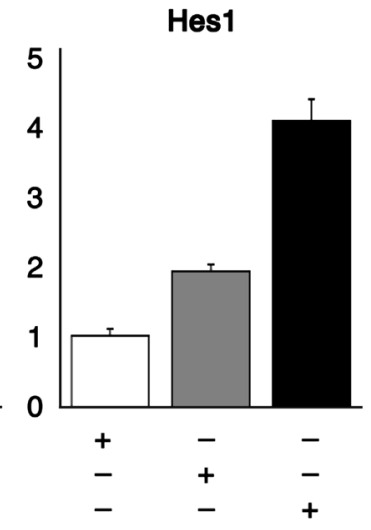

Figure 2

Quantitative analysis of $\mathrm{C} 2 \mathrm{C} 12$ differentiation. (A) Western blotting analysis of myosin expression in $\mathrm{C} 2 \mathrm{C} 12$ cells. (B) Morphometric analysis of myosin-positive cells. Results from differentiation experiments were analyzed by scoring the number of myosin-immunostained cells as a percentage of all DAPI-positive cells. (C) DBD-Foxo1-ADA reporter gene assays. We carried out reporter gene assays using the canonical Foxo1-responsive lgfbp1 promoter (left panel) and the Hes1 promoter (right panel) in cells cotransfected with Foxo1-ADA or DBD-Foxo1-ADA. Western blot (inset) demonstrates that expression levels of the 2 proteins are similar. An asterisk indicates $P<0.01$ by ANOVA.
$(6,35)$. We confirmed that this mutant is unable to bind DNA by measuring insulin-like growth factor-binding protein 1 (Igfbp1) promoter activity, a canonical Foxo1 target. Foxo1-ADA increased Igfbp 1 promoter activity by 10 -fold whereas DBD-Foxo1-ADA was unable to do so (Figure 2C). Surprisingly, this mutant was as effective as the DNA binding-competent Foxo1-ADA at inhibiting differentiation (Figure 1L). These data indicate that Foxo1 controls differentiation independently of its ability to bind DNA in a sequence-specific manner.

Foxo1 binds to Csl and is recruited to the Hes 1 promoter. Notch1-IC binds to and coactivates Csl to promote Hes and Hey expression (11). Based on the results with the DBD-Foxo1-ADA mutant, we determined whether Foxo1 interacts with Csl in a Notch-dependent manner using coculture of $\mathrm{C} 2 \mathrm{C} 12$ cells expressing Notch 1 receptor with HEK293 cells expressing the Notch ligand Jagged1 or LacZ as a negative control. We provide several lines of evidence that Foxo1 and Csl interact in cultured cells. We detected endogenous Foxo 1 in endogenous Csl immunoprecipitates, and the coimmunoprecipitation was significantly enhanced by activation of Notch signaling (Figure 3A). To confirm the specificity of the interaction, we expressed HA-tagged Foxo1 and FLAG-tagged Csl in C2C12 cells. Following immunoprecipitation with anti-HA (Foxo1) antiserum, we detected FLAG-Csl in immunoblots (Figure 3B). Conversely, following immunoprecipitation with anti-FLAG (Csl) antiserum, we detected HA-Foxo1 in immunoblots (Figure 3C). The ability to coimmunoprecipitate with Csl appears to be specific to Foxo1, as we failed to detect other Foxo isoforms in Csl immunoprecipitates (Supplemental Figure 6). A truncated Foxo1 mutant $(\Delta 256$, encod- ing aa 1-256) (36) retained the ability to interact with Csl. We detected FLAG-Csl in immunoprecipitates (Figure 3D) and HA- $\Delta 256$ in FLAG-Csl immunoprecipitates (Figure 3E), indicating that Csl interacts with the Foxo1 $\mathrm{N}$ terminal domain.

To determine whether this is a direct protein-protein interaction and map the interaction domain(s), we first carried out pull-down assays with affinity-purified glutathione-S-transferase-Foxo1 (GSTFoxo1) produced in bacteria and FLAG-Csl expressed in HEK293 cells. We detected Csl association with full-length and $\mathrm{N}$ terminal Foxo1 (aa 1-300) but not with C terminal Foxo1 (aa 290-655) or GST (Figure 4A). We next mapped the Csl domain that interacts with Foxo 1 using a cell-free system with GST-Foxo1 and GSTFlag-Csl purified from bacterial cultures. Again, we recovered fulllength (aa 1-655) and $N$ terminal (aa 1-300) but not $C$ terminal (aa 290-655) Foxo1 in Csl immunoprecipitates. Conversely, N terminal Foxo1 interacts with $\mathrm{N}$ terminal Csl (Figure 4B).

We used Csl deletion mutants to map the Foxo1-binding domain in Csl. These studies indicate that Foxo1 binds to a domain encompassing aa 172-279 (Figure 4C), which is contained within the Csl $\mathrm{NH}_{2}$ terminal domain (NTD) domain (37) (Figure 4C). Interestingly, this domain is required for DNA and corepressor binding but does not contribute to Notch binding $(38,39)$.

Csl binds to a consensus sequence in the Hes 1 promoter (40), which thus provides a useful readout assay of the Foxo/Csl interaction. If the latter were required to regulate $\mathrm{C} 2 \mathrm{C} 12$ differentiation, 3 predicted conditions should be met: (a) Foxo1 should be detected in chromatin immunoprecipitation (ChIP) assays spanning the Csl element in the Hes1 promoter; (b) the interaction should be 
A

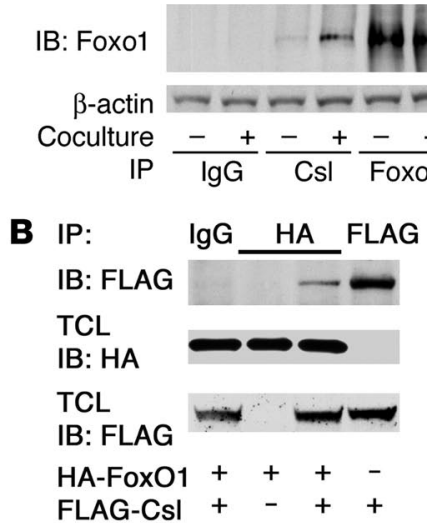

D IP:

IgG Myc FLAG

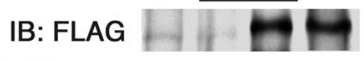

TCL

IB: Myc

TCL

IB: FLAG

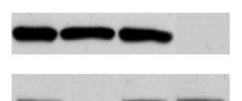

Myc- $\Delta 256+++-$

FLAG-CsI + - + +
C IP:

IB: HA

$\lg$ FLAG HA

TCL

IB: HA

TCL

IB: FLAG

$\mathrm{HA}-\mathrm{FoxO} 1+-++$

FLAG-CsI +++-

E IP:

IB: HA

TCL

IB: HA

TCL

IB: FLAG

$H A-\triangle 256$

IgG FLAG HA

FLAG-CsI +-++

differentiation dependent; and (c) inhibition of differentiation by Foxo1-ADA should be accompanied by constitutive binding to the Csl element in the Hes1 promoter. Figure 4D demonstrates that all predictions are fulfilled. First, we performed ChIP assays using

\section{Figure 3}

Foxo1 coimmunoprecipitates with Csl. (A) Coimmunoprecipitation of endogenous Foxo1 and Csl in $\mathrm{C} 2 \mathrm{C} 12$ cells cocultured with LacZexpressing (denoted by the minus sign) or Jagged1-expressing HEK293 cells (denoted by the plus sign). (B and C) Coimmunoprecipitation experiments in $\mathrm{C} 2 \mathrm{C} 12$ cells cotransfected with FLAG-CsI and HA-Foxo1. (D and E) Coimmunoprecipitation experiments in $\mathrm{C} 2 \mathrm{C} 12$ cells cotransfected with FLAG-Csl and the truncated mutant Myc- or HA-tagged $\Delta 256$ Foxo1. TCL, total cellular lysate.

primers spanning the Csl-binding site of Hes 1 in differentiating C2C12 cells. We detected endogenous Foxo1, Notch1, and Csl in immunoprecipitates from undifferentiated cells (Figure 4D). As the PCR-amplified sequence contains no forkhead binding sites, we concluded that Foxo1 binds to this DNA fragment via Csl. Moreover, binding of both Foxo 1 and Notch 1 decreased as cells became differentiated (days 1 and 2). When we transduced cells with constitutively nuclear Foxo1-ADA, differentiation was inhibited (Figure 1C) and the mutant Foxo1 was persistently bound to the Hes1 promoter, as were Csl and Notch1 (Figure 4D).

We next analyzed Hes 1 expression. The prediction was that Hes 1 levels should correlate with occupancy of the Hes 1 promoter by Foxo1 and Notch1. Indeed, Hes1 mRNA expression declined as Foxo 1 and Notch 1 binding to Csl decreased while myosin protein levels increased (Figure 4D). To rule out a direct effect of Foxo1 on Csl transcription, we carried out reporter gene assays with the Csl promoter. Foxo 1 failed to activate expression of a Csl reporter gene despite the presence of 10 repeats of a forkhead binding site in the Csl promoter (ref. 41 and data not shown). Moreover, Csl expression was unaffected in $\mathrm{C} 2 \mathrm{C} 12$

\section{Figure 4}

Foxo1 binds directly to CsI. (A) GST pull-down assays of GST-Foxo1 fusion protein with Csl immunoprecipitated from HEK293 cells. (B and C) Binding of GST-Foxo1 and GST-FLAG-Csl in a cell-free system and mapping of the Csl interaction domain. Full-length and truncated fragments of GSTFoxo1 and GST-FLAG-CsI were purified from bacteria and coincubated. Thereafter, Csl was isolated using anti-FLAG antibody, and the immunoprecipitate was analyzed by immunoblotting with anti-Foxo1 or anti-FLAG antibodies. (D) Hes1 promoter ChIP assay spanning the Csl-binding site in $\mathrm{C} 2 \mathrm{C} 12$ cells to detect endogenous Fox01, Csl, and Notch1 (Endog) or following transduction with Foxo1-ADA during myoblast differentiation. Input represents DNA extracted from chromatin prior to immunoprecipitation. Hes1 (semiquantitative RT-PCR) and myosin (Western blot) expression corresponding to each time point are shown. Day 0 is defined as the time when cells were serum deprived to induce myoblast fusion.
A

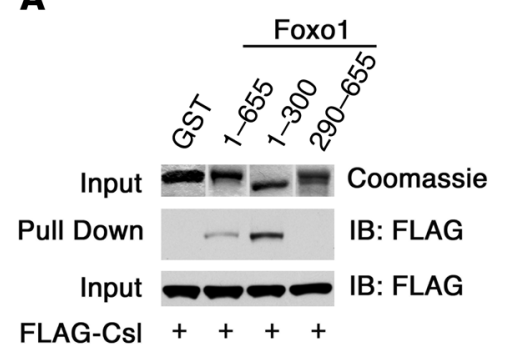

C

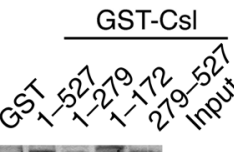

Input 무를

Coomassie

IB: Foxo1
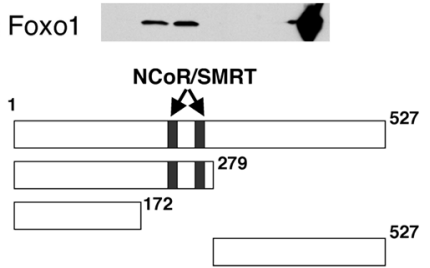

B
GST-Flag-Csl

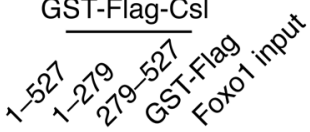

IP: FLAG HHI || Foxo1 1-655

IB: Foxo1 HGIIITFoxo1 1-300

IP: FLAG -

IB: FLAG

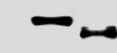

D
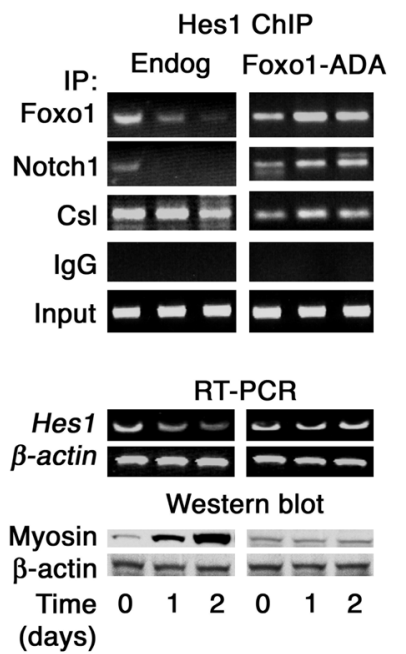
A

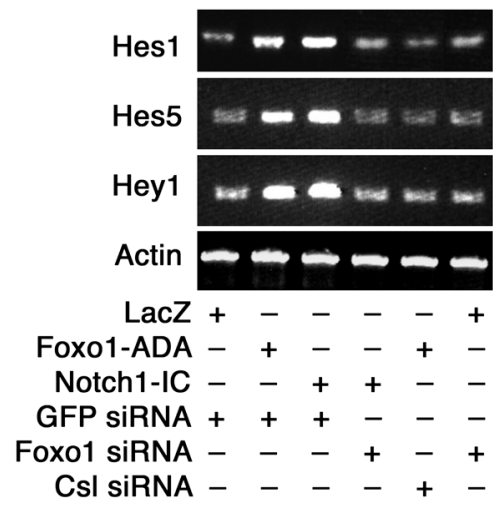

B Hes1 reporter activity

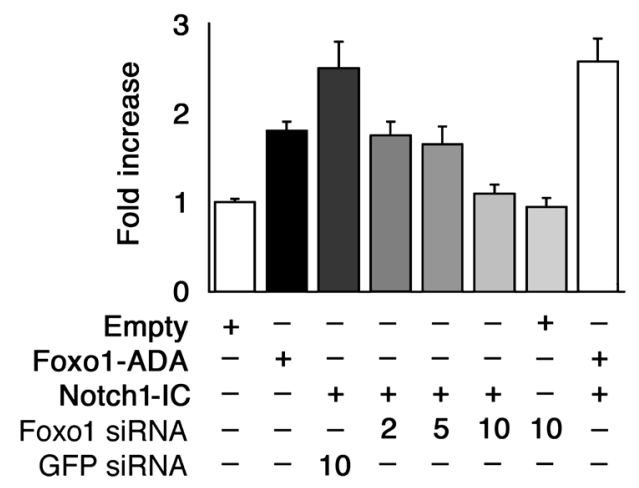

cells expressing Foxo1-ADA (data not shown). These data indicate that Foxo1 regulates Notch-dependent differentiation via protein/protein interactions with Csl.

Foxo1 is required for Notch induction of Hes and Hey genes via Csl. We examined the ability of Foxo1-ADA to promote expression of endogenous Hes1, Hes5, and Hey1 in C2C12 cells. Both Foxo1ADA and Notch1-IC increased the expression of the 3 genes while Foxo1 siRNA inhibited Hes1, Hes5, and Hey1 expression induced by Notch1-IC (Figure 5A). Foxo1 siRNA had no effect on Hes1, Hes5, and Hey 1 expression in growth factor-deprived cells (Figure 5A).

We focused the next set of experiments on Hes1, as a prototypical Notch target gene. We tested Foxo1's ability to regulate Hes1 transcription using reporter assays with the Hes1 promoter as well as measurements of Hes 1 expression. Foxo1-ADA and Notch1-IC induced Hes 1 promoter activity by 1.8 - and 2.5 -fold, respectively. Cotransfection of Foxo1-ADA with Notch1-IC caused a 2.5-fold increase (Figure 5B). Cotransfection of Foxo1 siRNA suppressed Notch-induced Hes1 activity in a dose-dependent manner while control siRNA had no effect (Figure 5B). We obtained similar results with a synthetic Hes 1 reporter containing 4 tandem repeats of the Csl-binding motif (Supplemental Figure 7). Moreover, DBDFoxo1-ADA was able to induce Hes1 reporter gene activity to an even greater extent than Foxo1-ADA, confirming that direct DNA binding is not required for Foxo1 activation of Hes 1 (Figure 2C).

The failure of Notch1-IC to induce Hes1 expression in cells expressing Foxo 1 siRNA suggests that Foxo 1 is required for Csl/ Notch interaction. Thus, we investigated the binding of Foxo1 and Notch 1 to the Hes 1 promoter in a coculture system. We cocultured C2C12 cells expressing Notch1 with HEK293 cells expressing the Notch ligand Jagged 1 to induce activation of endogenous

\section{Figure 5}

Foxo1 regulates Notch-induced Hes1, Hes5, and Hey1 expression. (A) Hes 1, Hes5, and Hey1 expression measured by semiquantitative RT-PCR in C2C12 cells transduced with Fox01-ADA or Notch1-IC following transfection of GFP, Foxo1, or CsI siRNA as indicated. (B) Hes1 reporter gene assays in HEK293 cells transduced with Foxo1-ADA, Notch1-IC, Foxo1 siRNA, GFP siRNA, or control plasmid (empty). We measured luciferase activity and normalized it by $\beta$-galactosidase activity. The data represent arbitrary units relative to control empty vector.

Notch signaling. Coculture in the presence of Jagged1-expressing cells increased endogenous Foxo1 (Figure 6A) and Notch1 binding to the Hes1 promoter in ChIP assays (Figure 6, A and B) (42). These data are consistent with the observation that Foxo1 coimmunoprecipitation with Csl increased upon coculture (Figure 3A). To determine whether Foxo1 binding to the Hes1 promoter is Csl dependent, we inhibited Csl expression with siRNA (Supplemental Figure 8). Transfection of Csl siRNA inhibited both Foxo1 and Notch 1 binding to Hes 1 promoter (Figure 6A), indicating that they are Csl dependent. Moreover, Foxo1-ADA failed to induce Hes 1 expression in the presence of Csl siRNA (Figure 5A). The results of ChIP experiments were corroborated by Hes 1 promoter assays. Expression of Jagged 1 or Notch 1 alone had no effect on Hes 1 activity, but coculturing yielded a 3.7-fold increase in Hes 1 reporter gene activity (Figure 6C). Foxo1 siRNA abolished Notch binding to the Hes 1 promoter in ChIP assays (Figure 6B) and induction of Hes 1 promoter activity (Figure 6C). These results suggest that Foxo1 is required for binding of Notch 1 to the Hes 1 promoter and provide a mechanism whereby inhibition of Foxo1 expression restores differentiation of myoblasts expressing Notch1-IC. The ability of Foxo1 siRNA to inhibit Notch induction of Hes 1 in a coculture system rules out the possibility that the effects observed in differentiation experiments with Notch1-IC are due to nonphysiologic activation of Notch signaling by the truncated intracellular Notch1 mutant (15).

Foxo1 promotes corepressor clearance and Maml1 binding to Csl. To clarify the molecular mechanism of Foxo1-dependent activation of Hes1 expression, we investigated corepressor/coactivator exchange at the Hes 1 promoter. Activation of Notch cleared the corepressors nuclear corepressor (NcoR) and silencing mediator for retinoid and thyroid hormone receptor (Smrt) (43) and recruited the coactivator mastermind-like 1 (Maml1) (42) to the Hes1 promoter. Foxo1 siRNA prevented Notch-induced corepressor exchange (Figure 6D). These data are consistent with the observation that Foxo 1 binds to the region 172-279 of Csl (Figure 4C), which has been shown to contain the NcoR/Smrt binding sites $(38,39)$.

To demonstrate that the observed changes in the transcriptional complex result in changes in Hes 1 activity, we investigated expression of Hes 1 target genes involved in myogenesis. Hes 1 has been proposed to suppress myoblast differentiation by inhibiting the basic helix-loop-helix transcription factor MyoD without affecting Myf5 $(16,17)$. Expression analyses revealed that Notch1-IC or Foxo1-ADA suppressed MyoD, while $M y f 5$ was unaffected. Notch decoy or Foxo1 siRNA partly restored $M y o D$ expression (Figure 6E).

Altered fiber type composition in skeletal muscle lacking Foxo1. Based on the cellular data, we undertook to probe Foxo1 function in muscle differentiation in vivo using conditional gene inactivation. The predicted outcome of this experiment was accelerated differentiation of MyoD-containing but not Myf5-containing 
A

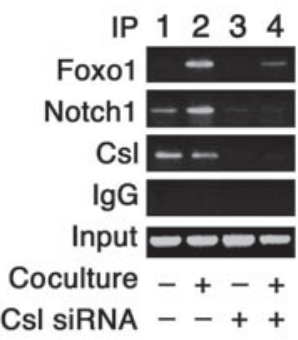

B
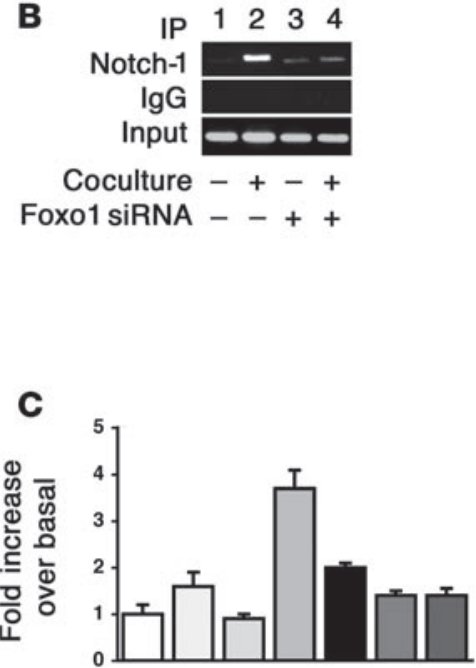

Empty vector +----Jagged1 -+-++++ Notch1 - -+++++ GFP siRNA - - - $10---$ Foxo1 siRNA - - - - 510 Notch decoy,-----+
D

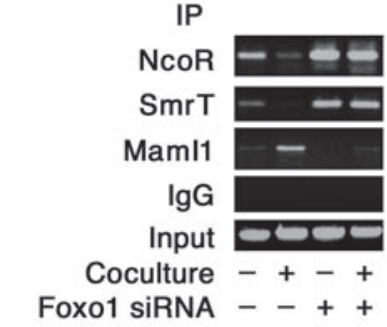

$\mathbf{E}$

\begin{tabular}{|c|c|c|c|c|c|c|}
\hline MyoD & & - & - & - & - & - \\
\hline Myf5 & - & - & - & - & & - \\
\hline Actin & $\sim$ & $\sim$ & $\sim$ & $\sim$ & $\sim$ & $\sim$ \\
\hline Induction & - & + & + & + & + & + \\
\hline LacZ & + & + & - & - & - & - \\
\hline $0 \times 01-A D A$ & - & - & + & + & - & - \\
\hline Notch1-IC & - & - & - & - & + & + \\
\hline d & - & - & - & + & - & - \\
\hline siRN & - & - & - & - & - & \\
\hline
\end{tabular}

$\mathbf{F}$

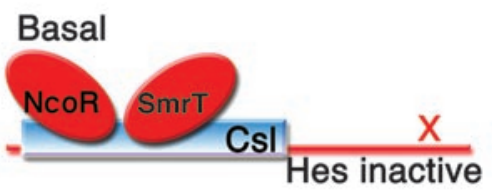

\section{Figure 6}

Foxo1 is required for Notch binding to the Hes1 promoter and activation of Hes1 target genes. (A) ChIP assays of endogenous Foxo1 and Notch1 in C2C12 cells cocultured with LacZexpressing (denoted by a minus sign) or Jagged1-expressing HEK293 cells (denoted by a plus sign) in the absence (lanes 1 and 2) and presence (lanes 3 and 4 ) of CsI siRNA. (B) ChIP assays of endogenous Notch1 in coculture system in the absence (lanes 1 and 2) and presence (lanes 3 and 4 ) of Foxo1 siRNA. (C) Hes1 promoter assays following coculture in the absence and presence of Foxo1 or GFP siRNA. (D) ChIP assays of NcoR and Smrt and Maml1 binding to Hes 1 in the coculture system in the absence (lanes 1 and 2) and presence (lanes 3 and 4) of Foxo1 siRNA. (E) Expression of MyoD, Myf5, and $\beta$-actin in $\mathrm{C} 2 \mathrm{C} 12$ cells by semiquantitative RT-PCR. (F) Model of Foxo1 and Notch regulation of Hes1 promoter. myoblasts. Because MyoD is the predominant myogenic factor in fast fibers while myogenin is the predominant factor in slow fibers (44), the removal of Foxo/Notch inhibition on MyoD expression should result in increased formation of fast fibers, potentially at the expense of slow fibers.

There are 3 Foxo isoforms in mice: Foxo1, Foxo3, and Foxo 4 $(8,9)$. The latter is predominant in most muscle types $(45)$ except soleus, where Foxo 1 is the most abundant (Figure 7A). Coincidentally, soleus is also physiologically enriched in slow-twitch fibers and thus allowed us to readily test our hypothesis. We inactivated Foxo 1 expression in skeletal muscle by crossing mice homozygous for a floxed Foxo1 allele with myogenin-cre transgenics. mRNA analysis indicated that the knockout occurred as planned (data not shown). Histological analyses revealed a reduction of type I (slowtwitch) fibers in soleus of myogenin-Foxo1 (Myog-Foxo) mice while type II fiber-enriched muscles were unaffected (Figure 7B). Consistent with the histological findings, expression of type I fiber markers decreased while type II fiber markers increased in MyogFoxo1 mice (Figure 7C). We then analyzed expression of the myogenic transcription factors MyoD, Myf5, and myogenin. MyoD is the predominant factor in fast fibers and myogenin in slow fibers (44). Consistent with the histopathology, we found a 2-fold increase in MyoD expression and an approximately $80 \%$ decrease in myogenin while Myf5 expression was unchanged (Figure 7C). Moreover, expression of the Foxo1 coactivator Pgc1 $\alpha$, which regu- lates type I fiber determination (30) was unchanged, indicating that the phenotype of Myog-Foxo1 mice cannot be accounted for by decreased Foxo1-dependent Pgc1 $\alpha$ transcription (Figure 7C) (46). As a functional correlate of the observed fiber type switch, we examined running performance on a treadmill. Indeed, MyogFoxo1 mice displayed reduced running capacity, as predicted from the reduction in type I (endurance) fibers (Figure 7D).

Finally, to determine whether these changes reflected developmental alterations in fiber-type specification as opposed to adaptive or cell-nonautonomous factors, we determined MyoD expression in Foxo1 (24) and Notch1 knockout (25) embryos at E9.5. In Foxo1 $1^{-/-}$embryos, MyoD levels increased $3.1 \pm 1.1$-fold, and in Notch $1^{-/}$embryos $7.3 \pm 2.9$-fold compared with controls $(P<0.05$ in both mutants versus wild type, $n=4)$. The increase in MyoD expression observed in vivo is consistent with the physical and functional interactions between Foxo 1 and Notch at this key signaling nexus in myoblast differentiation. Thus, we propose that the fiber-type switch in Myog-Foxo1 mice is the result of accelerated differentiation of MyoD-containing myoblasts during embryonic development.

\section{Discussion}

This study provides biochemical, cellular, and genetic evidence that Foxo and Notch pathways cooperate in the regulation of muscle differentiation. The data reveal what we believe is a novel mode of Foxo 1 action to promote corepressor exchange at the Hes 1 
A

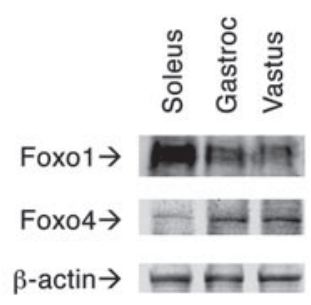

C

Slow-twitch markers

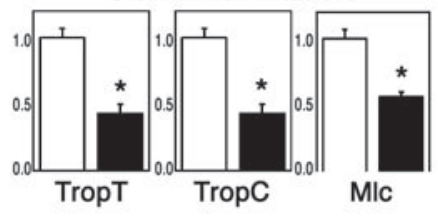

Fast-twitch markers

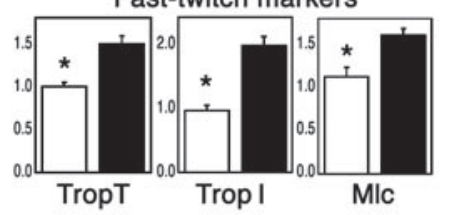

Muscle differentiation markers

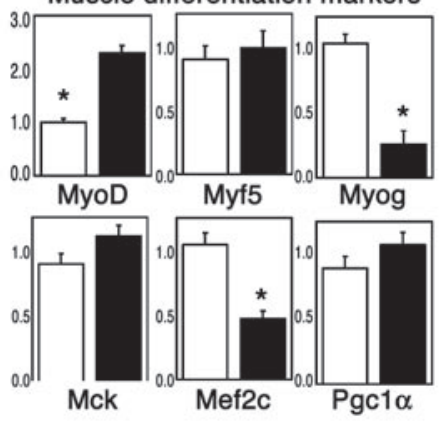

B
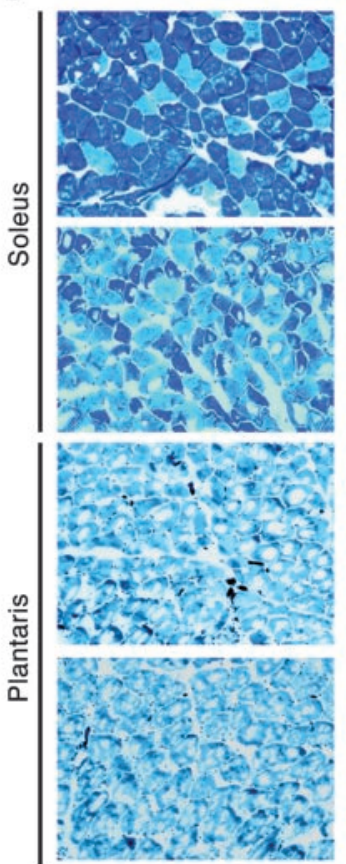

Metachromatic stain
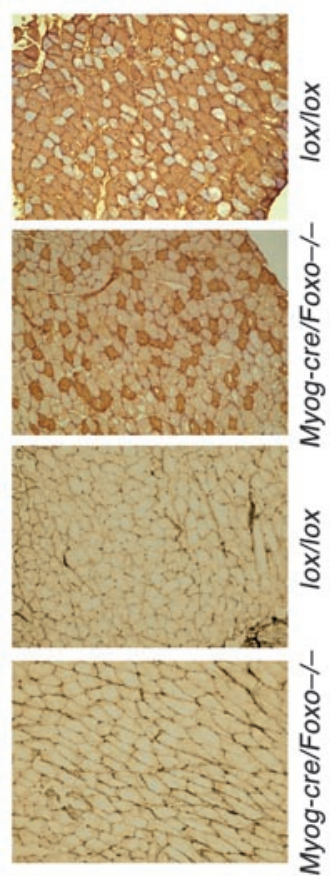

Anti-slow myosin

D

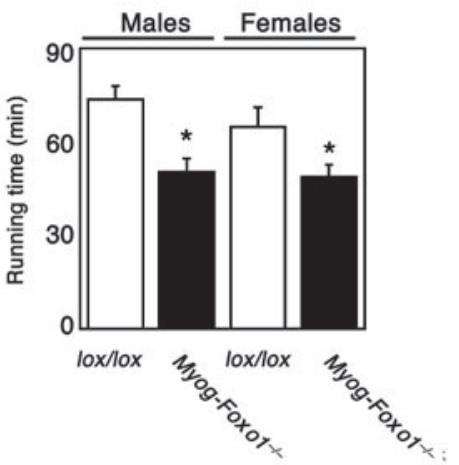

Figure 7

Conditional ablation of Foxo1 in skeletal muscle. (A) Western blot analysis of Foxo1 and Foxo4 expression levels in various muscle types. Gastroc, gastrocnemius muscle; Vastus, vastus lateralis muscle. (B) Metachromatic and immunohistochemical analysis of soleus and plantaris muscle from Myog-Foxo1 mice and control (lox/lox) littermates. Original magnification, $\times 10$. (C) Gene expression analysis of Myog-Foxo1 (black bars) and control mice (white bars); TropC, troponin-C; TropT, troponin-T; Mlc, myosin light chain; Myog, myogenin; Mck, muscle-type creatine kinase. Data are means \pm SEM of 3 independent measurements ( $n=6$ for each genotype). An asterisk indicates $P<0.05$ by ANOVA. (D) Treadmill performance test in 8-week-old Myog-Foxo1 mice and lox/lox littermates ( $n=6$ for each genotype). An asterisk indicates $P<0.05$ by ANOVA. promoter via direct binding to the Csl NTD region (Figure 6F). We propose that Foxo1 binding to this domain stabilizes the Notch/Csl complex and promotes corepressor clearance and Maml1 recruitment, consistent with the proposed role of NTD from structural studies (37). The findings also provide a mechanism by which 2 major biochemical pathways, the phosphoinositol 3-kinase/Akt pathway and the Notch/Hes pathway, converge in a synergistic manner to control cellular differentiation in vivo.

The proposed role for Foxo1 is independent of its transcriptional function and involves a direct interaction with Csl. While our studies have focused on Hes1 as a prototypical effector of Notch1 signaling, our data should not be construed to indicate that Hes 1 is the sole mediator of the Notch/Foxo interaction. For example, we have observed a similar Foxo/Notch epistasis in the differentiation of preadipocytes, PC-12, and HUVECs, suggesting that Foxo interacts with Notch in multiple cell contexts (data not shown). We propose that Notch/Foxo cooperation integrates environmental cues through Notch with metabolic cues through Foxo1 to regulate progenitor cell maintenance and differentiation. This 2 -tiered mechanism allows committed progenitor cells in various tissues to avoid differentiation in response to developmental cues
(Notch) when Foxo1 is active, i.e., in the absence of growth factors. These cells would then persist in a dormant state in adult tissues, where they can terminally differentiate in response to a combination of Notch ligand and hormonal/nutritional cues leading to Foxo1 inhibition. This interpretation is consistent with the fibertype switch observed in Foxo1-deficient muscle, an observation that appears to position Foxo 1 as a fate decider within the myogenic lineage, as opposed to an inducer of the myogenic program. It remains to be seen whether other Foxo and Notch isoforms also interact and how they contribute to this process.

The demonstration that Foxo 1 is a coregulator of gene expression provides a potential explanation for the protean functions of this transcription factor. Interesting questions emerging from our studies involve how the switch from one function to the other is effected and how the complex posttranslational modifications of Foxo1 in response to growth factors, hormones, and nutrients impinge on this process. The findings have broad implications for the pathophysiology of disease processes that involve Foxo1 signaling. A potential implication of our observation is the ability to explore the use of agents that inhibit Notch signaling (47) as a treatment of metabolic disorders characterized by excessive Foxo function (48). 


\section{Methods}

Animal generation and analysis. Myogenin-cre (49) and Foxo $1^{\text {flox }}$ mice have been described (9). The wild-type, null, and Foxo $1^{\text {flox }}$ alleles were detected using PCR with primers 5'-GCTTAGAGCAGAGATGTTCTCACATT-3', 5'-CCAGAGTCTTTGTATCAGGCAAATAA-3', and 5'-CAAGTCCATTAATTCAGCACATTGA-3'. Prior to the treadmill performance test, mice were trained for 2 days (Columbus Instruments). The test was performed at $15 \mathrm{~m} / \mathrm{min}$ for the first 30 minutes, followed by $1 \mathrm{~m} / \mathrm{min}$ increases at $10 \mathrm{~min}$ ute intervals until exhaustion. Skeletal muscle samples were quickly frozen in OCT matrix, and $7-\mu \mathrm{m}$ serial sections were obtained. Muscle fibers were typed using metachromatic ATPase (50) or immunostaining with anti-skeletal slow myosin (Sigma-Aldrich). For embryonic studies, we set up timed matings of heterozygous Foxo1 (24) or Notch1 (25) mice and recovered embryos at E9.5. mRNA was isolated from whole embryos, and real-time RT-PCR was performed as described below. All animal experiments were approved by the Columbia University Animal Care and Utilization Committee.

Viral expression studies. C2C12 cells were differentiated as described $(3,4)$. Foxo1-ADA, Notch1-IC, Jagged1, Csl, and Notch decoy adenoviral and mammalian expression vectors have been described $(36,51)$. We generated retroviruses expressing Foxo1-ADA and Notch1-IC using the PQCXIH vector (Clontech). To generate Notch decoy (pAdlox Notch1ECD-Fc), the extracellular domain of Notch1 (bp 241-4229, GenBank accession number $\mathrm{X} 57405$ ) was fused in frame with human IgG Fc tag and cloned into pAdlox. Retroviral supernatant was produced from cells transiently cotransfected with $\mathrm{pVSV}-\mathrm{G}$ vector (Clontech) and designated PQCXIH vector into GP2-293 cells (BD Biosciences). To generate the DNA binding-deficient Foxo1, we replaced N208 and H212 with alanine and arginine, respectively, using QuikChange Mutagenesis Kit (Stratagene). The mutations were then cloned in the backbone of the Foxo1-ADA mutant.

Luciferase assay and coculture assay. We transfected HEK293 cells with Hes1luciferase (-194 to 160 from transcription start site) (Hes1/pGL2 basic [Stratagene]), synthetic Hes1-luciferase (containing a $4 \times$ Csl binding site, $4 \times \mathrm{Csl} / \mathrm{pGL} 2$ basic) or Csl-luciferase ( -1536 to 22 , Csl/pGL2 basic) reporter genes along with pCMV5, pCMV5-Foxo1-ADA, pQNC-Notch1-IC, pHyTc (51), Notch decoy, or Foxo1 siRNA. We used plasmid pRSV- $\beta$-galactosidase as a control of transfection efficiency (51). For coculture assay, we expressed Notch1 in C2C12 cells and Jagged 1 or LacZ in HEK293 cells by transfection. We then harvested HEK293 cells and seeded them on C2C12 cells. After 1 hour incubation, we used the cocultured cells for experiments.

Western blotting and immunoprecipitation. We performed these assays according to standard techniques using anti-myosin (MF-20), anti-HA (12CA5; Boehringer Mannheim), anti-FLAG (M2; Sigma-Aldrich), antiFoxo1 (H128 and N20; Santa Cruz Biotechnology Inc.), anti-Notch1 (C-20; Santa Cruz Biotechnology Inc.), anti-Csl (Millipore and Santa Cruz Biotechnology Inc.), anti-NcoR (Santa Cruz Biotechnology Inc.), anti-Smrt
(Santa Cruz Biotechnology Inc.), or anti-MAML1 (Millipore) antibodies. For Foxo/Csl coimmunoprecipitation, we used purified nuclear fractions (52). Because Csl migrates close to IgG heavy chain on SDS-PAGE, we used dimethylpyrimilidate (DMP; Pierce) to cross-link antibodies to protein A beads and avoid IgG contamination of eluted protein complexes (52).

ChIP assays. We performed ChIP assays in C2C12 cells as described previously (4) and in cocultured cells as described by Fryer (42). The primer pairs employed to amplify the Csl-binding site of the Hes 1 promoter are as follows: 5'-GCAAAGCCCAGAGGAAAGAGTTAG-3' and 5'-AGGAGAGAGGTAGACAGGGGATTC-3'.

siRNA transfection and siRNA-resistant Foxo1. The Foxo1-specific siRNA sequence is $5^{\prime}$-ACGGAGGATTGAACCAGTATA-3'. The Csl-specific siRNA sequence is $5^{\prime}$-TAGGGAAGCTATGCGAAATTA- ${ }^{\prime}$. siRNA was transfected using lipofectamine-plus reagent (Invitrogen). We generated siRNA-resistant Foxo 1 by replacing 3 residues (underlined) in the sequence 5 '-ACGGCGGTCTGAACCAGTATA-3'. Primer sequences employed for real-time RT-PCR studies are in the Supplemental Methods.

Recombinant proteins and interaction assays. We generated GST-FLAG-Csl encompassing aa 1-527, 1-279, 1-172, and 279-527 fragments by cloning into pGEX6P-1. GST-Foxo1 constructs have been described (53). Following bacterial culture and iso-propyl-thio-galactose induction, we purified GST fusion proteins and incubated them together. Thereafter, we isolated GST-FLAG/Csl by immunoprecipitation with anti-FLAG antibody, washed the immune pellets extensively, and performed immunoblot with anti-Foxo1 antiserum.

Statistics. All results are presented as \pm SEM. $P$ values were calculated by 1-factor ANOVA.

\section{Acknowledgments}

This work was supported by NIH grants DK57539, DK64819, 5 RO1 HL062454 (JK), the Columbia Diabetes and Endocrinology Research Center (DK63608), and the Berrie Program in Cell-Based Therapies at Columbia University. We thank E. Olson (University of Texas Southwestern) for Myog-cre mice and members of the Accili and Kitajewski laboratories for stimulating discussions.

Received for publication March 7, 2007, and accepted in revised form June 6, 2007.

Address correspondence to: Domenico Accili, Department of Medicine, Columbia University College of Physicians and Surgeons, 1150 St. Nicholas Avenue, New York, New York 10032, USA. Phone: (212) 851-5332; Fax: (212) 851-5331; E-mail: da230@columbia.edu.

T. Kitamura and Y.I. Kitamura contributed equally to this work.
1. Singec, I., Jandial, R., Crain, A., Nikkhah, G., and Snyder, E.Y. 2007. The leading edge of stem cell therapeutics. Annu. Rev. Med. 58:313-328.

2. Accili, D., and Arden, K.C. 2004. FoxOs at the crossroads of cellular metabolism, differentiation, and transformation. Cell. 117:421-426.

3. Hribal, M.L., Nakae, J., Kitamura, T., Shutter, J.R., and Accili, D. 2003. Regulation of insulin-like growth factor-dependent myoblast differentiation by Foxo forkhead transcription factors. J. Cell Biol. 162:535-541

4. Nakae, J., et al. 2003. The forkhead transcription factor foxo 1 regulates adipocyte differentiation. Dev. Cell. 4:119-129.

5. Potente, M., et al. 2005. Involvement of Foxo transcription factors in angiogenesis and postnatal neovascularization. J. Clin. Invest. 115:2382-2392. doi:10.1172/JCI23126.
6. Liu, Z.P., Wang, Z., Yanagisawa, H., and Olson, E.N. 2005. Phenotypic modulation of smooth muscle cells through interaction of Foxo 4 and myocardin. Dev. Cell. 9:261-270.

7. Castrillon, D.H., Miao, L., Kollipara, R., Horner, J.W., and DePinho, R.A. 2003. Suppression of ovarian follicle activation in mice by the transcription factor Foxo3a. Science. 301:215-218.

8. Tothova, Z., et al. 2007. FoxOs are critical mediators of hematopoietic stem cell resistance to physiologic oxidative stress. Cell. 128:325-339.

9. Paik, J.H., et al. 2007. Foxos are lineage-restricted redundant tumor suppressors and regulate endothelial cell homeostasis. Cell. 128:309-323.

10. Shawber, C.J., and Kitajewski, J. 2004. Notch function in the vasculature: insights from zebrafish, mouse and man. Bioessays. 26:225-234.

11. Lai, E.C. 2002. Keeping a good pathway down: transcriptional repression of Notch pathway target genes by CSL proteins. EMBO Rep. 3:840-845.

12. Jensen, J., et al. 2000. Control of endodermal endocrine development by Hes-1. Nat. Genet. 24:36-44.

13. Ross, D.A., Rao, P.K., and Kadesch, T. 2004. Dual roles for the Notch target gene Hes-1 in the differentiation of 3T3-L1 preadipocytes. Mol. Cell. Biol. 24:3505-3513.

14. Ohtsuka, T., et al. 1999. Hes 1 and Hes5 as notch effectors in mammalian neuronal differentiation. EMBO J. 18:2196-2207.

15. Shawber, C., et al. 1996. Notch signaling inhibits muscle cell differentiation through a CBF1-independent pathway. Development. 122:3765-3773.

16. Sasai, Y., Kageyama, R., Tagawa, Y., Shigemoto, R., and Nakanishi, S. 1992. Two mammalian helix-loop-helix factors structurally related to Drosophila hairy and Enhancer of split. Genes Dev. 
6:2620-2634.

17. Kuroda, K., et al. 1999. Delta-induced Notch signaling mediated by RBP-J inhibits MyoD expression and myogenesis. J. Biol. Chem. 274:7238-7244.

18. Nofziger, D., Miyamoto, A., Lyons, K.M., and Weinmaster, G. 1999. Notch signaling imposes two distinct blocks in the differentiation of $\mathrm{C} 2 \mathrm{C} 12$ myoblasts. Development. 126:1689-1702.

19. Wilson-Rawls, J., Molkentin, J.D., Black, B.L., and Olson, E.N. 1999. Activated notch inhibits myogenic activity of the MADS-Box transcription factor myocyte enhancer factor 2C. Mol. Cell. Biol. 19:2853-2862.

20. Hirsinger, E., et al. 2001. Notch signalling acts in postmitotic avian myogenic cells to control MyoD activation. Development. 128:107-116.

21. Conboy, I.M., and Rando, T.A. 2002. The regulation of Notch signaling controls satellite cell activation and cell fate determination in postnatal myogenesis. Dev. Cell. 3:397-409.

22. Shawber, C.J., Das, I., Francisco, E., and Kitajewski, J. 2003. Notch signaling in primary endothelial cells. Ann. N. Y. Acad. Sci. 995:162-170.

23. Limbourg, F.P., et al. 2005. Essential role of endothelial Notch 1 in angiogenesis. Circulation. 111:1826-1832.

24. Hosaka, T., et al. 2004. Disruption of forkhead transcription factor (FOXO) family members in mice reveals their functional diversification. Proc. Natl. Acad. Sci. U. S. A. 101:2975-2980.

25. Krebs, L.T., et al. 2000. Notch signaling is essential for vascular morphogenesis in mice. Genes Dev. 14:1343-1352.

26. McKinsey, T.A., Zhang, C.L., and Olson, E.N. 2001. Control of muscle development by dueling HATs and HDACs. Curr. Opin. Genet. Dev. 11:497-504.

27. Rudnicki, M.A., and Jaenisch, R. 1995. The MyoD family of transcription factors and skeletal myogenesis. Bioessays. 17:203-209.

28. Bassel-Duby, R., and Olson, E.N. 2006. Signaling pathways in skeletal muscle remodeling. Annu. Rev Biochem. 75:19-37.

29. Schiaffino, S., and Reggiani, C. 1996. Molecular diversity of myofibrillar proteins: gene regulation and functional significance. Physiol. Rev.
76:371-423.

30. Lin, J., et al. 2002. Transcriptional co-activator PGC-1 alpha drives the formation of slow-twitch muscle fibres. Nature. 418:797-801.

31. Lagouge, M., et al. 2006. Resveratrol improves mitochondrial function and protects against metabolic disease by activating SIRT1 and PGC-1alpha. Cell. 127:1109-1122.

32. Nickoloff, B.J., Osborne, B.A., and Miele, L. 2003. Notch signaling as a therapeutic target in cancer: a new approach to the development of cell fate modifying agents. Oncogene. 22:6598-6608.

33. Nickoloff, B.J., et al. 2002. Jagged-1 mediated activation of notch signaling induces complete maturation of human keratinocytes through NF-kap$\mathrm{paB}$ and PPARgamma. Cell Death Differ. 9:842-855.

34. Pan, Y., et al. 2004. gamma-secretase functions through Notch signaling to maintain skin appendages but is not required for their patterning or initial morphogenesis. Dev. Cell. 7:731-743.

35. Dowell, P., Otto, T.C., Adi, S., and Lane, M.D. 2003. Convergence of peroxisome proliferator-activated receptor gamma and Foxo1 signaling pathways. J. Biol. Chem. 278:45485-45491.

36. Nakae, J., Kitamura, T., Silver, D.L., and Accili, D. 2001. The forkhead transcription factor Foxo 1 (Fkhr) confers insulin sensitivity onto glucose-6phosphatase expression. J. Clin. Invest. 108:1359-1367. doi:10.1172/JCI200112876.

37. Kovall, R.A., and Hendrickson, W.A. 2004. Crystal structure of the nuclear effector of Notch signaling, CSL, bound to DNA. EMBO J. 23:3441-3451.

38. Hsieh, J.J., and Hayward, S.D. 1995. Masking of the CBF1/RBPJ kappa transcriptional repression domain by Epstein-Barr virus EBNA2. Science. 268:560-563.

39. Kao, H.Y., et al. 1998. A histone deacetylase corepressor complex regulates the Notch signal transduction pathway. Genes Dev. 12:2269-2277.

40. Tun, T., et al. 1994. Recognition sequence of a highly conserved DNA binding protein RBP-J kappa. Nucleic Acids Res. 22:965-971.

41. Kawaichi, M., et al. 1992. Genomic organization of mouse J kappa recombination signal binding protein (RBP-J kappa) gene. J. Biol. Chem.
267:4016-4022.

42. Fryer, C.J., White, J.B., and Jones, K.A. 2004. Mastermind recruits CycC:CDK8 to phosphorylate the Notch ICD and coordinate activation with turnover. Mol. Cell. 16:509-520.

43. Liang, Y., Chang, J., Lynch, S.J., Lukac, D.M., and Ganem, D. 2002. The lytic switch protein of KSHV activates gene expression via functional interaction with RBP-Jkappa (CSL), the target of the Notch signaling pathway. Genes Dev. 16:1977-1989.

44. Hughes, S.M., et al. 1993. Selective accumulation of MyoD and myogenin mRNAs in fast and slow adult skeletal muscle is controlled by innervation and hormones. Development. 118:1137-1147.

45. Kitamura, T., et al. 2002. The forkhead transcription factor Foxo1 links insulin signaling to $P d x 1$ regulation of pancreatic $\beta$ cell growth. J. Clin. Invest. 110:1839-1847. doi:10.1172/JCI200216857.

46. Daitoku, H., Yamagata, K., Matsuzaki, H., Hatta, M., and Fukamizu, A. 2003. Regulation of PGC-1 promoter activity by protein kinase $B$ and the forkhead transcription factor FKHR. Diabetes. 52:642-649.

47. Miele, L., Miao, H., and Nickoloff, B.J. 2006. NOTCH signaling as a novel cancer therapeutic target. Curr. Cancer Drug Targets. 6:313-323.

48. Accili, D. 2004. Lilly lecture 2003: the struggle for mastery in insulin action: from triumvirate to republic. Diabetes. 53:1633-1642.

49. Knapp, J.R., et al. 2006. Loss of myogenin in postnatal life leads to normal skeletal muscle but reduced body size. Development. 133:601-610.

50. Ogilvie, R.W., and Feeback, D.L. 1990. A metachromatic dye-ATPase method for the simultaneous identification of skeletal muscle fiber types I, IIA, IIB and IIC. Stain Technol. 65:231-241.

51. Das, I., et al. 2004. Notch oncoproteins depend on gamma-secretase/presenilin activity for processing and function. J. Biol. Chem. 279:30771-30780.

52. Chi, T., Yan, Z., Xue, Y., and Wang, W. 2004. Purification and functional analysis of the mammalian SWI/SNF-family of chromatin-remodeling complexes. Methods Enzymol. 377:299-316.

53. Puigserver, $P$., et al. 2003. Insulin-regulated hepatic gluconeogenesis through FOXO1-PGC-1alpha interaction. Nature. 423:550-555. 